\title{
CONHECIMENTO EM AVALIAÇÃO PSICOLÓGICA
}

\author{
KNOWLEDGE IN PSYCHOLOGICAL ASSESSMENT
}

\author{
Ana Paula Porto NORONHA ${ }^{1}$ \\ João Carlos ALCHIERI ${ }^{2}$
}

\begin{abstract}
RESUMO
O presente estudo teve como objetivo analisar o conhecimento de estudantes de psicologia a respeito de conteúdos relacionados à avaliação psicológica. Participaram 342 alunos de duas instituições de ensino do interior, sendo uma de São Paulo e outra do Rio Grande do Sul. Para a realização do estudo foi elaborado um inventário de 45 itens, com questões dicotômicas, com o objetivo de investigar o conceito de avaliação, o uso de instrumentos e a aprendizagem de testes. Os resultados indicaram que houve diferença entre as instituições e que sujeitos sem acesso aos conteúdos de avaliação tiveram desempenhos menores do que os alunos que já cursaram disciplinas relativas à avaliação.
\end{abstract}

Palavras-chave: Avaliação psicológica, testes psicológicos, ensino de avaliação.

\begin{abstract}
The present study had as aim to analyze the knowledge of psychology students regarding contents related to the psychological assessment. Participated 342 students, from an institution of education in the interior of the province of São Paulo and other of Rio Grande do Sul. For the accomplishment of the study a 45 items test was elaborated with dichotomy questions, whose objective was to investigate the concept of assessment, the use of tests and the learning of tests. The results have indicated that there was difference among the groups and that students without courses of psychological assessment have gotten better performances than the ones.

Key words: Psychological assessment, psychological tests, learning of assessment.

\footnotetext{
(1) Professora Doutora do Programa de Estudo Pós-Graduados em Psicologia da Universidade São Francisco. Endereço para correspondência: Rua: Alexandre Rodrigues Barbosa, 45 Centro - Itatiba/SP Cep: 13251-900. E-mail: ananoronha@saofrancisco.edu.br

(2) Doutorando em Psicologia do Desenvolvimento pela Universidade Federal do Rio Grande do Sul, Docente da Universidade do Vale do Rio dos Sinos.
} 


\section{INTRODUÇÃO}

A avaliação psicológica (AP) é um exame de caráter compreensivo efetuado para responder questões específicas quanto ao funcionamento psíquico adaptado ou não de uma pessoa durante um período específico de tempo ou para predizer o funcionamento psicológico da pessoa no futuro. A avaliação deve fornecer informações cientificamente fundamentadas tais que orientem, sugiram, sustentem o processo de tomada de decisão em algum contexto específico no qual a decisão precisa levar em consideração informações sobre o funcionamento psicológico.

A formação profissional em geral tem a responsabilidade de proporcionar uma formação crítica, que gere atuações seguras e que possa servir à sociedade da melhor forma possível (Ferreira, Almeida \& Soares, 2001). Sobre o ensino de avaliação psicológica entende-se que ele representa um dos pontos principais na formação em Psicologia, e deve contemplar diferentes disciplinas em diversas áreas de ação do psicólogo como teorias da personalidade, medida em psicologia, teorias psicopatológicas, dentre outras (Alchieri \& Bandeira, 2002).

Por muito tempo, essa temática foi considerada como sendo sinônimo de ensino de testes psicológicos, nos quais o professor tinha como função repassar os conhecimentos referentes ao manejo de instrumentos objetivos (ditos psicométricos) e projetivos. Para alguns cursos atualmente, essa é ainda uma realidade, especialmente aqueles que ainda incluem em seu currículo as disciplinas de técnicas de avaliação como únicas representantes da formação em avaliação psicológica.

Pensando em problematizar a questão da avaliação no ensino de Psicologia, elaborou-se uma base metodológica de esteio à formação em AP que visa amparar o ensino fundamentado em um triplo eixo: a medida psicológica, o uso dos instrumentos e a atividade profissional na avaliação. Nesse primeiro artigo será discutida a questão do uso de instrumentos, para contextualizar a questão e assim próximos trabalhos apresentarão uma aprofundada discussão valendo-se dos dados apresentados aqui. Acredita-se que, desta maneira, será possível começar a leitura por qualquer um dos três tópicos e o leitor poderá ter a discussão contextualizada.

\section{ENSINANDO TESTES}

O ensino de testes foi um dos pontos de base para a discussão da atividade profissional nos currículos de muitos cursos no Brasil e, sem dúvida, aquele que apresentou maior variabilidade. Desde a lei 4119 referente à criação dos cursos de psicologia, a questão dos testes já estava contemplada nas disciplinas de Técnicas de Exame Psicológico, as TEPs como eram coloquialmente chamadas. Uma vez que seu caráter era obrigatório, estavam elas divididas ao longo de um vasto currículo nos cursos do país. Assim, os instrumentos eram de escolha dos professores que ministravam as disciplinas, ou da direção do curso quanto à possibilidade de encontrar docentes que sabiam testes. Esta cultura dos testes lecionados caracterizava-se como "(...) uma prática a ser aprendida e reproduzida, perpetuava-se mecanicamente no ensino das universidades que multiplicavam a Psicologia pelo país" (Alchieri \& Bandeira, 2002, p.3).

O ensino estava, então, respaldado pela seqüência interminável dos testes ensinados em sucessivos semestres cuja ênfase era o conhecimento da aplicação, da correção e da interpretação dos resultados. Ensinados nesta sucessão, o que menos importava era identificar condições de uso ou limitação dos instrumentos, o critério de escolha dos testes, a ausência de disciplinas que fundamentavam a medida psicológica, as teorias sobre personalidade e demais áreas avaliadas.

Os professores cuja experiência estava sustentada na utilização prática dos testes e assim, saber usar e aplicar um determinado teste era freqüentemente condição de ingresso na docência. As disciplinas de TEPs foram então as portas de entrada para muitos psicólogos poderem passar a ser docentes. 
Com o quadro instalado, a discussão do que se podia ou não ensinar, como ensinar, quem poderia ensinar foi transpondo os muros da academia e chegando às plenárias de eventos científicos da área. Primeiramente, um grupo no Conselho Regional de Psicologia do RS $\left(7^{\mathrm{a}}\right.$ Região) formou a Comissão de Métodos e Técnicas Psicológicas e com ela, em 1986, organizou um evento, único no país até então, o I Encontro sobre Testes Psicológicos do CRP 07 no Rio Grande do Sul (Alves, Alchieri \& Marques, 2002). Com o objetivo de discutir basicamente o ensino de testes, o evento transformou-se, em pouco mais de três edições, num evento nacional de referência para a área e, em 1999, ganhou um caráter internacional. Outros eventos, nacionais e internacionais, representaram o mesmo anseio de discutir questões sobre testes psicológicos, geralmente associados com o tipo de instrumento, versões, cursos sobre testes e pesquisas na área. Em comum todos tratavam do descaso da categoria em relação ao instrumental, à falta de normas nacionais e à ausência de um maior cuidado por parte dos órgãos de classe com o uso indiscriminado dos testes.

Um certo número de referências hoje pode ser encontrado e respalda, assim, a origem e a caracterização das preocupações em relação aos trabalhos versando sobre o ensino das Técnicas de Exame Psicológico (Custódio \& Duarte, 1986, Calejon \& Vistué, 1994, Lima, 1994, Rocha Jr., 1994, Bueno, 1995, Jacquemin, 1995, Custódio, 1995, Schwartz, 1996, Rocha Jr. \& Castro, 1997, Souza, 1997, Bromberg, 1997, Avoglia, 1997, Avoglia, Castro \& Custódio, 1999, Gomes \& Noffs, 1999, Alves, 2000, Castro, 2001, Calejon \& Santos, 2001, Lima, 1999, 2001, Alchieri \& Cruz, 2001, Alchieri \& Bandeira, 2002).

Em que pese a importância da temática sobre o quê, como e quando ensinar, poucos trabalhos evidenciaram a questão partindo do ponto de vista dos instrumentos utilizados, de maneira a assegurar a representação do que realmente acontece no ensino de instrumentos e quais eram eles (Jacquemin, 1995). Assim, os dados mais específicos sobre os instrumentos utilizados no ensino em distintas regiões do país puderam ser apresentados por Bandeira e cols (1995), Castro (1998), Pacheco e Takahashi (1999), Alves (2000) e Alves, Alchieri e Marques (2001). Um dos trabalhos pioneiros quanto à busca de sinais de identificação e caracterização das vicissitudes do uso de instrumentos no ensino e a percepção por parte dos docentes pode ser visto em Noronha (1999).

Também cabe a consideração do número de instrumentos em uso no país e suas principais características técnicas. Até pouco tempo (Alchieri, 2000), não existia um levantamento fidedigno sobre o número de instrumentos em uso no Brasil e, assim, a questão ficava pouco embasada quanto à verdadeira extensão da problemática.

Sabe-se que o número de testes comercializados no Brasil não ultrapassa duas centenas (mais especificamente 176, em verificação realizada até janeiro de 2003), sendo que destes, 80\% não apresentam as mínimas condições de uso cientificamente asseguradas para estarem no mercado editorial. A análise das características técnicas (validade, precisão e padronização) está sendo objeto de investigação por parte de um projeto (Noronha, Alchieri \& Primi, 2001) com apoio da FAPESP, além de representar um dos objetos de trabalho do Conselho Federal de Psicologia (CFP, 2001, 2003).

Atualmente a situação brasileira em AP é muito diferente do observado nas primeiras visões críticas das décadas de setenta e oitenta. Longe de termos uma carência de artigos e livros, estamos com uma das maiores produções na área da América Latina, existem mais sociedades e instituições científicas na área com representação nacional, o número de dissertações e teses sobre a questão da AP permite que se preveja uma massa crítica de pesquisadores e docentes capazes de conduzir pesquisa de ponta 
na área e que com isto, o número de instrumentos e técnicas psicológicas tenha um correspondente de qualidade comparável e de nível internacional. Contudo, como está o ensino de avaliação psicológica, representado pelos instrumentos ensinados nos cursos de graduação?

A pergunta parte do pressuposto que há uma distinção entre o que é ensinado na prática profissional e o que é desenvolvido e pesquisado no Brasil atualmente. Uma das razões se fundamenta nos dados apresentados por Bandeira e cols. (1995) e em um recente trabalho (Alchieri, Parastchuck \& Cruz, 2001) quanto ao ensino de testes em universidades da região sul do país. Observou-se nos instrumentos ensinados, no período de cinco anos, a ausência de mudança e renovação dos testes, ou seja, parece que a prática docente não se mostrou capaz de absorver a produção nacional e se atualizar a partir dela.

A realização do presente estudo baseouse nas necessidades da área de avaliação psicológica e teve como objeto de trabalho a análise do conhecimento de AP de estudantes de dois cursos de Psicologia brasileiros.

\section{MÉTODO}

\section{Participantes}

Participaram do presente estudo 322 sujeitos de cursos de psicologia de duas instituições, sendo uma do Estado de São Paulo e outra do Rio Grande do Sul. A idade média foi de 24 anos (DP 7,6), variando entre 17 e 61 anos, sendo que, quanto ao gênero, $89,4 \%(F=288)$ eram do sexo feminino e $10,2 \%(F=33)$ do sexo masculino. Um sujeito não preencheu o dado, portanto não fez parte dessa análise.

Os sujeitos que cursavam o período matutino corresponderam a 46,6\% ( $F=150)$, enquanto $44,7 \%(F=144)$ estudavam no noturno e 4,3\% ( $F=14)$ no período integral. Alguns sujeitos não preencheram o dado (4,3\%). No que se refere às disciplinas de avaliação psicológica, vale ressaltar que $41 \%(F=132)$ dos sujeitos já as cursaram e 59\% ( $F=190)$ não as fizeram.
Os participantes foram organizados em dois grupos, a saber:

G1: 180 estudantes do Estado de São Paulo;

G2: 142 estudantes do Estado do Rio Grande do Sul.

\section{Material}

Para o desenvolvimento do presente estudo foi elaborado um teste de conhecimentos de Avaliação Psicológica, contendo 45 questões com respostas dicotômicas (verdadeiro ou falso). Os itens do instrumento abordavam questões sobre o conceito de avaliação psicológica, sobre aprendizagem e sobre o uso de instrumentos. A construção ficou sob responsabilidade da primeira autora; dois especialistas na área fizeram a análise de juízes.

\section{Procedimento}

Após a devida autorização para a realização da pesquisa, o instrumento foi aplicado coletivamente em sala de aula dos cursos de psicologia de duas instituições. Em seguida, os dados foram organizados e lançados numa planilha eletrônica para análise e elaboração dos resultados.

\section{RESULTADOS}

Os itens do instrumento foram escritos tendo em vista quatro áreas de conhecimento, a saber: conceito de avaliação $(1,4,10,12,13,24$, $26,27,30$ e 39$)$, conceito de instrumento de avaliação $(5,7,14,15,16,18,20,21,22,25,29$ e 42), uso de instrumento $(2,3,9,19,31,34,35$, 36 e 38) e aprendizagem de instrumentos (6, 8, $11,17,23,28,32,33,37,40,41,43,44$ e 45$)$. As análises que se seguem podem ser reunidas em três eixos: itens com maior número de respostas em branco (missings), porcentagem de acertos dos itens em cada grupo e no total e comparação de médias dos grupos. 
Tabela 1. Respostas dos sujeitos dos dois grupos em cada item.

\begin{tabular}{|c|c|c|c|c|c|c|}
\hline \multirow{2}{*}{ Itens } & \multicolumn{2}{|c|}{ SP } & \multicolumn{2}{|c|}{ RS } & \multirow{2}{*}{ Total } & \multirow{2}{*}{$p$} \\
\hline & $\mathrm{V}$ & $\mathrm{F}$ & $\mathrm{V}$ & $\mathrm{F}$ & & \\
\hline 1 & 180 & 0 & 141 & 1 & 322 & 0,259 \\
\hline 2 & 169 & 9 & 136 & 6 & 320 & 0,727 \\
\hline 3 & 52 & 127 & 24 & 118 & 321 & $0,011^{*}$ \\
\hline 4 & 20 & 159 & 13 & 128 & 320 & 0,568 \\
\hline 5 & 23 & 157 & 15 & 127 & 322 & 0,541 \\
\hline 6 & 131 & 46 & 107 & 35 & 319 & 0,785 \\
\hline 7 & 102 & 78 & 128 & 14 & 322 & 0,093 \\
\hline 8 & 5 & 175 & 9 & 133 & 322 & 0,120 \\
\hline 9 & 61 & 113 & 54 & 86 & 314 & 0,521 \\
\hline 10 & 104 & 73 & 86 & 54 & 317 & 0,630 \\
\hline 11 & 27 & 153 & 39 & 103 & 322 & $0,006^{*}$ \\
\hline 12 & 165 & 15 & 114 & 28 & 322 & $0,003^{*}$ \\
\hline 13 & 142 & 32 & 121 & 19 & 314 & 0,250 \\
\hline 14 & 88 & 91 & 71 & 71 & 321 & 0,881 \\
\hline 15 & 101 & 77 & 94 & 46 & 318 & 0,059 \\
\hline 16 & 149 & 30 & 120 & 19 & 318 & 0,449 \\
\hline 17 & 157 & 23 & 126 & 16 & 322 & 0,680 \\
\hline 18 & 41 & 136 & 62 & 76 & 315 & 0,000 ** \\
\hline 19 & 43 & 137 & 23 & 119 & 322 & 0,090 \\
\hline 20 & 169 & 11 & 136 & 6 & 322 & 0,452 \\
\hline 21 & 9 & 170 & 17 & 125 & 321 & $0,024^{*}$ \\
\hline 22 & 158 & 22 & 118 & 24 & 322 & 0,234 \\
\hline 23 & 61 & 115 & 76 & 65 & 317 & $0,001^{* *}$ \\
\hline 24 & 97 & 76 & 97 & 41 & 311 & $0,010^{*}$ \\
\hline 25 & 150 & 27 & 118 & 24 & 319 & 0,690 \\
\hline 26 & 51 & 125 & 32 & 108 & 316 & 0,219 \\
\hline 27 & 134 & 39 & 106 & 30 & 309 & 0,919 \\
\hline 28 & 98 & 80 & 77 & 62 & 317 & 0,952 \\
\hline 29 & 153 & 25 & 124 & 15 & 318 & 0,466 \\
\hline 30 & 99 & 79 & 84 & 58 & 320 & 0,525 \\
\hline 31 & 124 & 54 & 109 & 31 & 318 & 0,101 \\
\hline 32 & 104 & 76 & 78 & 64 & 322 & 0,609 \\
\hline 33 & 50 & 130 & 24 & 118 & 322 & $0,021^{*}$ \\
\hline 34 & 173 & 6 & 141 & 1 & 321 & 0,107 \\
\hline 35 & 162 & 18 & 128 & 12 & 320 & 0,664 \\
\hline 36 & 100 & 79 & 92 & 48 & 319 & 0,075 \\
\hline 37 & 55 & 125 & 40 & 102 & 322 & 0,641 \\
\hline 38 & 20 & 160 & 12 & 129 & 321 & 0,440 \\
\hline 39 & 15 & 165 & 11 & 131 & 322 & 0,848 \\
\hline 40 & 22 & 157 & 7 & 135 & 321 & $0,022^{*}$ \\
\hline 41 & 44 & 135 & 19 & 123 & 321 & $0,012^{*}$ \\
\hline 42 & 11 & 168 & 17 & 124 & 321 & 0,120 \\
\hline 43 & 159 & 19 & 106 & 33 & 317 & $0,002^{*}$ \\
\hline 44 & 39 & 140 & 13 & 129 & 321 & $0,002^{*}$ \\
\hline 45 & 7 & 172 & 1 & 141 & 321 & 0,067 \\
\hline
\end{tabular}

(*) significativo ao nível de 0,05

$\left.{ }^{* *}\right)$ significativo ao nível de 0,001. 
No que se refere aos missings, vale ressaltar que das 45 questões, apenas 31,1\% delas $(F=14)$ foram respondidas por todos os sujeitos. Das restantes, sete tiveram mais respostas em branco do que as demais, sendo elas $9,10,18,23,24,26$ e 43, que abordam respectivamente o uso de instrumentos (em uma avaliação o psicólogo usa de 5 a 10 instrumentos de avaliação); o conceito de avaliação (uma avaliação psicológica é factual, ou seja, representa um momento específico da vida do indivíduo; Psicometria é a ciência que estuda a medida psicológica; a realização de avaliações psicológicas é recente na Psicologia); o conceito de instrumento (no Brasil existem tantos testes psicológicos quanto nos países mais desenvolvidos); e a aprendizagem (um elemento que dificulta a aprendizagem de testes é quando o conteúdo é discutido logo no início do curso; penso que se deve aprender mais testes na faculdade). Na Tabela 1 é possível encontrar detalhadamente a quantidade de respostas (verdadeiro/falso) emitidas pelos sujeitos de cada grupo, assim como no total.

Considerando as diferenças de resposta entre cada grupo, a aplicação do qui-quadrado revelou que, em 7 itens, a diferença foi significativa (Tabela 1), a saber: 9, 10, 18, 23, 24, 26 e 43.

A Tabela 2 apresenta a porcentagem de respostas corretas em cada item dadas pelos sujeitos no total e em cada grupo. Os itens que tiveram as melhores porcentagens de acerto foram: item 1 - a avaliação psicológica é uma atividade importante para o profissional de Psicologia; item 2 - a avaliação psicológica pode ser utilizada em muitos contextos de atuação profissional; item 7 - os testes devem ser válidos; item 8 - a aprendizagem de testes deveria acontecer apenas no último ano do curso; item 20 - a entrevista e a observação são técnicas de avaliação; item 21 - os testes psicológicos são instrumentos pouco importantes na prática profissional do psicólogo; item 34 - para usar um teste o profissional deve ter domínio do instrumento; item 35 - alguns psicólogos usam
Tabela 2. Porcentagem de acerto dos grupos em cada item e no total.

\begin{tabular}{|c|c|c|c|}
\hline Item & SP & RS & Total \\
\hline 1 & 100 & 99,3 & 99,7 \\
\hline 2 & 94,9 & 95,8 & 95,3 \\
\hline 3 & 70,1 & 3,1 & 76,3 \\
\hline 4 & 88,8 & 90,8 & 89,7 \\
\hline 5 & 87,2 & 89,4 & 88,2 \\
\hline 6 & 74,0 & 75,4 & 74,6 \\
\hline 7 & 56,7 & 90,1 & 92,9 \\
\hline 8 & 97,2 & 93,7 & 95,7 \\
\hline 9 & 64,9 & 61,4 & 63,4 \\
\hline 10 & 58,8 & 61,4 & 59,9 \\
\hline 11 & 85,0 & 72,5 & 79,5 \\
\hline 12 & 91,7 & 80,3 & 86,6 \\
\hline 13 & 81,6 & 86,4 & 83,8 \\
\hline 14 & 50,8 & 50,0 & 50,5 \\
\hline 15 & 56,7 & 67,1 & 61,3 \\
\hline 16 & 83,2 & 86,3 & 84,6 \\
\hline 17 & 87,2 & 88,7 & 87,9 \\
\hline 18 & 76,8 & 55,1 & 67,3 \\
\hline 19 & 76,1 & 83,8 & 79,5 \\
\hline 20 & 93,9 & 95,8 & 94,7 \\
\hline 21 & 95,0 & 88,0 & 91,9 \\
\hline 22 & 87,8 & 83,1 & 85,7 \\
\hline 23 & 34,7 & 53,9 & 43,2 \\
\hline 24 & 56,1 & 70,3 & 62,4 \\
\hline 25 & 84,7 & 83,1 & 84,0 \\
\hline 26 & 71,0 & 77,1 & 73,7 \\
\hline 27 & 77,5 & 77,9 & 77,7 \\
\hline 28 & 55,1 & 55,4 & 55,2 \\
\hline 29 & 85,5 & 89,2 & 87,1 \\
\hline 30 & 69,7 & 59,2 & 57,2 \\
\hline 31 & 69,7 & 77,9 & 73,3 \\
\hline 32 & 42,2 & 45,1 & 43,5 \\
\hline 33 & 72,2 & 83,1 & 77,0 \\
\hline 34 & 96,7 & 99,3 & 97,8 \\
\hline 35 & 85,7 & 91,4 & 90,6 \\
\hline 36 & 44,1 & 34,3 & 39,8 \\
\hline 37 & 88,9 & 71,8 & 70,5 \\
\hline 38 & 88,9 & 91,5 & 90,0 \\
\hline 39 & 8,3 & 7,8 & 8,1 \\
\hline 40 & 71,0 & 95,1 & 91,0 \\
\hline 41 & 93,9 & 86,6 & 80,4 \\
\hline 42 & 93,3 & 88,0 & 91,0 \\
\hline 43 & 78,7 & 89,3 & 83,6 \\
\hline 44 & 78,2 & 90,9 & 83,8 \\
\hline 45 & 96,9 & 99,3 & 97,5 \\
\hline
\end{tabular}


testes sem o devido cuidado; item 38 - outros profissionais podem usar qualquer tipo de teste psicológico; item 40 - a aprendizagem de um teste é suficiente para generalizar conhecimentos para os demais instrumentos; item 42-o psicólogo não deve usar testes, pois eles reduzem o homem a números; e item 45 - os testes não servem para nada.

Já no que se refere aos itens com menor freqüência de acerto, destaque deve ser dado aos itens 10,14, 23, 28, 30, 32 e 36 que tratam, respectivamente, das seguintes questões: uma avaliação psicológica é factual, ou seja, representa um momento específico da vida do indivíduo (10); os testes eventualmente devem ser precisos (14); um elemento que dificulta a aprendizagem de testes é quando o conteúdo é discutido logo no início do curso (23); a aprendizagem de testes é dificultada pela leitura de manuais de testes escritos em inglês (28); a avaliação é concebida como uma atividade exclusiva do psicólogo (30); os testes são realmente aprendidos na prática clínica do psicólogo (32); e há orientações precisas sobre o uso de testes em âmbito nacional e internacional (36).

Ainda no que diz respeito à análise dos acertos/erros de cada grupo de sujeitos, os resultados indicaram que o $\mathrm{G} 2$ obteve desempenhos mais altos que o $\mathrm{G} 1 \mathrm{em} 64,4 \%$ dos itens, enquanto o G1 obteve melhor desempenho em 35,6\%, embora a realização do teste $t$ de Student tenha revelado que a diferença entre as médias dos grupos não é significativa $(t=-, 515 ; p=0,608)$.

\section{DISCUSSÃO}

Inicialmente observou-se que na amostra prevalecem algumas das principais características quanto aos dados sociodemográficos dos acadêmicos em Psicologia no Brasil, quanto ao número de participantes do sexo feminino e quanto à caracterização quanto aos aspectos da diversidade de níveis nos cursos. Esta representação é muito importante enquanto possibilidade de se compreender os resultados deste trabalho e de demonstrar a concepção identificada de avaliação psicológica.

As diferenças apontadas quanto aos três grupos de resultados apresentados, instrumentos conceitos de medida e de instrumentais e a aprendizagem associada destes, podem ser tomadas como representativas de um ponto comum quanto ao desconhecimento dos objetivos principais da avaliação, de suas características, da realidade brasileira (Aribi, \& Cavalini, 1999), e quanto aos processos envolvidos no ensino nas instituições dos dois Estados. Não se procurou avaliar nestas expressões uma quantidade de conhecimento dominado ou a interpretação das respostas quanto a uma análise de testes, mas sim um aspecto crucial, o posicionamento crítico dos acadêmicos (Pereira \& Carellos, 1995) diante das informações obtidas nas disciplinas de avaliação psicológica. Todos estes pontos refletem invariavelmente a expressão que o ensino adquire, especialmente de avaliação psicológica, num estudante de graduação que se posiciona diante de uma prática profissional (Souza, 1997).

Se tomados os resultados expressos na primeira tabela quanto às respostas com maiores percentuais de acerto, podemos verificar que estes dados permitem caracterizar quatro categorias de representação com seus respectivos itens: os aspectos profissionais (itens 1 - a avaliação psicológica é uma atividade importante para o profissional de Psicologia; item 2 - a avaliação psicológica pode ser utilizada em muitos contextos de atuação profissional); os aspectos técnicos (item 7 - os testes devem ser válidos; item 20 - a entrevista e a observação são técnicas de avaliação; item 34 - para usar um teste o profissional deve ter domínio do instrumento; item 35 - alguns psicólogos usam testes sem o devido cuidado); de ensino e formação profissional (item 8 - a aprendizagem de testes deveria acontecer apenas no último ano do curso; item 40 - a aprendizagem de um teste é suficiente para generalizar conhecimentos para os demais instrumentos); e percepção crítica da atividade (item 38 - outros profissionais podem usar qualquer tipo de teste psicológico; item 42 - o psicólogo não deve usar testes, pois eles 
reduzem o homem a números e item 45 - os testes não servem para nada; item 21 - os testes psicológicos são instrumentos pouco importantes na prática profissional do psicólogo).

É importante destacar que, dentre as opções, a escolha dos itens pode refletir uma percepção técnica e profissional distorcida quanto à aplicabilidade dos instrumentos psicológicos, mais precisamente distorcida pela representação que os respondentes têm da avaliação. Estas idéias se representam às opiniões dos sujeitos, acadêmicos de Psicologia, mostram um posicionamento técnico nada calcado na realidade e, principalmente, ideológico a questão do instrumento, sendo a avaliação uma extensão deste e não sua origem. Seguindo com a idéia, pode-se considerar que os professores de avaliação (Vasconcelos \& Toledo de Santana, 2001) seguem ensinando avaliação psicológica ou ensinando os testes psicológicos?

É possível que a representação do ensino seja embasada pela dos instrumentos e assim, conseqüentemente, a lógica discursiva das questões marque sua expressão num plano ideológico da crítica, aliás, já muito desatualizada e ultrapassada. Cabe salientar que estes questionamentos perpassam o curso do início ao fim, sem que os novos dados das disciplinas de avaliação, em geral expressos nas aulas de testes antigos, sejam removidos, como se evidenciou em outros estudos (Alves, Alchieri \& Marques, 2001).

O terceiro aspecto da análise das questões foi baseado na freqüência de erro e representa aqueles aspectos que menos caracterizam uma percepção certamente mais atualizada do acadêmico. Verificou-se que, para os respondentes, a necessidade de confrontar os aspectos citados foi dificultada certamente pela ausência de dados mais específicos da realidade da avaliação pelo acadêmico. As informações deveriam estar presentes no seu dia-a-dia, mas devido à falta destes informes durante o processo de ensino é identificada a lacuna de formação e tomada a baixa freqüência de acertos.

Outros aspectos podem ser relacionados como considerações adicionais ao presente estudo em sua análise desses resultados. Em primeiro lugar, a questão de a avaliação psicológica, com sua expressiva produção nacional, especialmente nos últimos anos, não estar contemplada nos currículos das disciplinas ministradas nas universidades. Desta forma, ensina-se mediante o uso de clássicos materiais (testes, livros e, não raro, o uso de apostilas apócrifas de professores). E, em segundo lugar, o desconhecimento quanto publicação de artigos e sua baixa freqüência vinculada à bibliografia das disciplinas; assim, seu conhecimento torna-se domínio de poucos professores e a atualidade deste conhecimento permanece representada como decorrente de eventos.

\section{CONCLUSÃO}

É possível que os aspectos principais do ensino de avaliação psicológica tenham de ser repensados e definidos com mais clareza nos novos currículos dos cursos de Psicologia do país. No entanto, se for mantida a e ênfase na caracterização da avaliação como uma atividade decorrente dos instrumentos ensinados e de suas idiossincrasias, certamente esta forma não irá ser eficaz na orientação do processo para além do quadro atual.

A necessidade de rever as questões do ensino leva autores como Guzzo (2001) a afirmar que o aprendizado das técnicas do exame psicológico isolado e pontual não assegura necessariamente o estabelecimento das competências necessárias, a expressão de habilidades como a conclusão de indicativos e a elaboração de resultados como laudos. Se, por um lado, a sociedade constantemente manifesta seu interesse no trabalho investigativo e avaliativo de aspectos psicológicos, por outro lado, no meio institucional a psicologia não consegue reconhecer sua expressiva potencialidade. Como se vê, ainda há muito a ser modificado, no que se refere à formação profissional como um todo, ao ensino de avaliação, em especial, e às técnicas propriamente ditas. 


\section{REFERÊNCIAS BIBLIOGRÁFICAS}

ALCHIERI, J. C. \& Bandeira, D. R. (2002). Ensino da Avaliação Psicológica noBrasil. Em R. Primi (Org.). Temas em Avaliação Psicológica (pp.35-39). Campinas: Impressão Digital do Brasil Gráfica e Editora Ltda.

ALCHIERI, J.C. (2000). Base de dados sobre os instrumentos psicológicos no Brasil. Trabalho apresentado na XXI Reunião da Associação Nacional de Pesquisa e Pós-Graduação em Psicologia, Serra Negra, SP.

ALCHIERI, J.C. \& Cruz, R.M. (2001) Recursos técnicos e estratégias utilizadas no ensino da avaliação psicológica nas universidades da região sul do Brasil. Anais do $28^{\circ}$ Congresso Interamericano de Psicologia. CD-ROM.

ALMEIDA, L. S. (1999). Avaliação Psicológica: exigências e desenvolvimentos nos seus métodos. Em S. M. Wechsler \& R. S. L. Guzzo (Orgs.). Avaliação psicológica-perspectiva internacional (pp.41-55). São Paulo: Casa do Psicólogo.

ALVES, I. C. B. (2002). Instrumentos disponíveis no Brasil para avaliação da inteligência. Em $R$. Primi (Org.). Temas em Avaliação Psicológica (pp. 80-102). Campinas: Impressão Digital do Brasil Gráfica e Editora Ltda.

ALVES, I. C. B., Alchieri, J. C. \& Marques, K. (2001). Panorama geral do ensino das técnicas de exame psicológico no Brasil. I Congresso de Psicologia Clínica - Programas e Resumos. Universidade Presbiteriana Mackenzie, São Paulo, 10-11.

ALVES, I.C.B. (2000). As atividades práticas no ensino das Técnicas de Exame Psicológico. Anais do VIII Simpósio Brasileiro de Pesquisa e Intercâmbio Científico, Águas de Lindóia/ SP, 171.

ARIBI, N. V., \& Cavalini, S. F. S. (1999). Testes de nível intelectual: como e por quê ensinálos. Anais do VIII Congresso Nacional de Avaliação Psicológica. Porto Alegre/RS, p. 62-68.

AVOGLIA, H.R.C. (1997). Observação Participativa como Estratégia de Ensino de Técnicas de Exame Psicológico. Boletim de Psicologia, XLVII, 107, 107-110.

AVOGLIA, H.R.C., Castro, D.S. \& Custódio, E.M. (1999). Avaliação Psicológica e Psicologia Social: relato de uma experiência de ensino interdisciplinar. Programa e Pôsteres. VIII Congresso Nacional de Avaliação Psicológica. Desafios para um Novo Milênio, 61.

BANDEIRA, D.R., Argimon, I.L., Alchieri, J.C., Oliveira, M.S. \& Werlang, B.G. (s/d). O Ensino de Testes Psicológicos nas Universidades do Rio Grande do Sul. Texto não publicado.

BROMBERG, M.H.P.F. (1997). O Ensino das Técnicas de Exame Psicológico: Aspectos Práticos e Metodológicos ou de Quando o Sujeito passa a ser Cliente. Boletim de Psicologia, XLVII, 107, 101-105.

Bueno, R.M.G. (1995). Ensino e Pesquisa. Boletim de Psicologia, XLV,102, 23-25.

CALEJON, L.M. \& Santos, M.R.M. (2001). Ensino de Testes psicológicos e a formação do psicólogo. Anais do IV Encontro da Sociedade Brasileira de Rorschach e outras Técnicas de Avaliação Psicológica, 164.

CALEJON, L.M. \& Vistué, A. (1994). Contribuição do Ensino de TEAP para Construção da Identidade Profissional do Psicólogo. Programa e Resumos. I Encontro de Técnicas de Exame Psicológico: Ensino, Pesquisa e Aplicações, 2.

CASTRO, P.F. (1998). O Ensino de Rorschach em uma Amostra Brasileira. Psicologia, Ciência e Profissão, 21 (1), 46-53.

CASTRO, P.F. (2001). Reflexões acerca do ensino e aprendizagem do Rorschach na realidade brasileira. Anais do IV Encontro da Sociedade Brasileira de Rorschach e outras Técnicas de Avaliação Psicológica, Itatiba/SP, 59-60.

CUSTÓDIO, E.M. \& Duarte, W.F. (1986). O Ensino de Técnicas de Exame Psicológico nos Cursos de Psicologia. Suplemento de Ciência e Cultura, 38 (7), 1055. 
CUSTÓDIO, E.M. (1995). O Ensino das Técnicas de Exame Psicológico. Boletim de Psicologia, XLV, 102, 27-34.

GOMES, I.C. \& Noffs, M.H. (1999). O Ensino de Técnicas Projetivas Gráficas no curso de Psicologia em duas instituições, particular e pública. PSIC - Revista da Vetor Editora, 1(1), 18-29.

GUZZO, R. S. L. (2001). Laudo psicológico: a expressão da competência profissional. Em L. Pasquali (Org.). Técnicas do Exame Psicológico - TEP. Volume I: Fundamentos das Técnicas Psicológicas (155-170). São Paulo: Casa do Psicólogo.

JACQUEMIN, A. (1995). Ensino e Pesquisa sobre Testes Psicológicos. Boletim de Psicologia, XLV,102, 19-21.

LIMA, R.A. (1999). O Reflexo das Atividades Práticas no Processo de Ensino-Aprendizagem das Técnicas de Exame Psicológico. Programa e Resumos do II Encontro sobre Psicologia Clínica, 76-77.

LIMA, R.A. (2001). O Reflexo das Atividades Práticas no Processo de Ensino-Aprendizagem das Técnicas de Exame Psicológico. Psicologia: Teoria e Pesquisa, 3 (2), 55-69.

NORONHA, A. P. P. (1999). Avaliação psicológica: usos e problemas com ênfase nos testes. Tese de Doutorado. Instituto de Psicologia, Pontifícia Universidade Católica de Campinas, 154p.

PACHECO, A.L.P.B. \& Takahashi, F. (1999). Avaliação Psicológica e sua Instrumentalização na Formação do Psicólogo. Programa e Pôsteres. VIII Congresso Nacional de Avaliação Psicológica. Desafios para um Novo Milênio, 42.
PEREIRA, A. P. C., \& Carellos, S. D. M. S. (1995). Examinando o Ensino das Técnicas de Exame Psicológico. Cadernos de Psicologia, 3(4), 33-36.

PRIMI, R. \& Munhóz, A. M. H. (1998). Um estudo sobre proficiência na disciplina de Técnicas de Avaliação Psicológica (TEP). PSICO-USF, 3 (2), 75-86.

ROCHA Jr. A. (1994). A Importância das Atividades Práticas para o Aprendizado de Técnicas de Exame Psicológico. Programa e Resumos. I Encontro de Técnicas de Exame Psicológico: Ensino, Pesquisa e Aplicações, 3.

ROCHA Jr. A. \& Castro, P. F. (1997). A Importância das Atividades Práticas no Ensino de Técnicas de Exame Psicológico. Anais. VII Encontro Nacional sobre Testes Psicológicos e I Congresso Ibero-Americano de Avaliação Psicológica, 288.

SCHWARZ L. R. (1996). A Importância da Vivência na Aprendizagem das Técnicas Projetivas de Personalidade. Programa e Resumos. II Encontro de Técnicas de Exame Psicológico: Ensino, Pesquisa e Aplicações, 58.

SOUZA, A. S. L. (1997). O ensino das cadeiras de Técnicas do Exame Psicológico. Boletim de Psicologia, v. XLVII n.107 jul/dez, 95-99.

VASCONCELOS, Z. B. \& Toledo de Santana, C. M. (2001). O Ensino das Técnicas de Exame Psicológico na Universidade Federal da Paraíba. Anais do I Congresso de Psicologia Clínica. Universidade Presbiteriana Mackenzie, São Paulo, 207-208.

Recebido para publicação em 17 de junho de 2003 e aceito em 2 de fevereiro de 2004. 